\title{
Immunolocalization of a Recombinant Cellulase in Transgenic Tobacco Plants
}

\author{
H. J. Bae*, H. Chamberland*, S. Laberge**, and Y. S. Kim*** \\ * Laval University, Ste-foy, Quebec, Canada G1K 7P4 \\ **Centre de Recherches et de Agriculture et Agroalimentaire Canada G1V 2J3 \\ ***Chonnam National University, Kwangju, Korea 500-757
}

Cellulose is the main constituent of plant and is an important commercial material with potential for degradation into various products including ethanol (1). Conversion of plant biomass to fermentable sugars is carried out using large -scale fermentation with commercial enzymes. The production cost of these enzymes remains however a significant barrier to the widespread utilization of this process. The use of genetically engineered plants ap pears as a more economic approach for large -scale production of proteins. Expression of different cellulase genes in transgenic plant has already been demonstrated $(2,3)$.

In the present work we have determined the localization of a recombinant cellulas $\mathrm{e}$ in tobacco plants. The gene is a modified version of the endoglucanase EGIV from Ruminococcus albus containing the cellulose binding domain CBDII from Clostridium sterdcorarium. Two different constructions were used with and without the chloroplast trans it peptide. By immunofluorescence microscopy we observed a strong labeling in the chloroplasts from plant transformed with the construct containing the transit peptide. On the contrary, in plants transformed without the transit peptide, the green fluoresce nce was mainly distributed in the cytoplasmic area as confirmed by double fluorescence confocal microscopy. Further investigations performed at the electron microscopy level showed the presence of numerous gold particles in the chloroplasts of tobacco tran sgenic line containing the transit peptide (Fig. A). In contrast, gold particles were more numerous over the cytosol in plants containing the gene construct without the transit peptide (Fig. B).

\section{References:}

1. Lynd, L. R., J. H. Cushman, R. J. Nichols, and C. H. Wyman. 1991. Fuel ethanol from cellulosic biomass. Science. 251:1318-1323.

2. Austin, S., E. T. Bingham, D. E. Mathews, M. N. Shahan, J. Will and R. R. Burgess. 1995. Production and field performance of transgenic alfalfa (Medicago sativa L.) expressing alpha-amylase and manganese-dependent lignin peroxidase. Euphytica 85: 381-393.

3. Ziegelhoffer, T., J. Will and S. Austin-Phillips. 1999. Expression of bacterial cellulases genes in transgenic alfalfa (Medicago Sativa L.), potato (Solanum tuberosum L.) and tobacco (Nicotiana tabacum L.) Mol. Breed. 5: 309-318. 

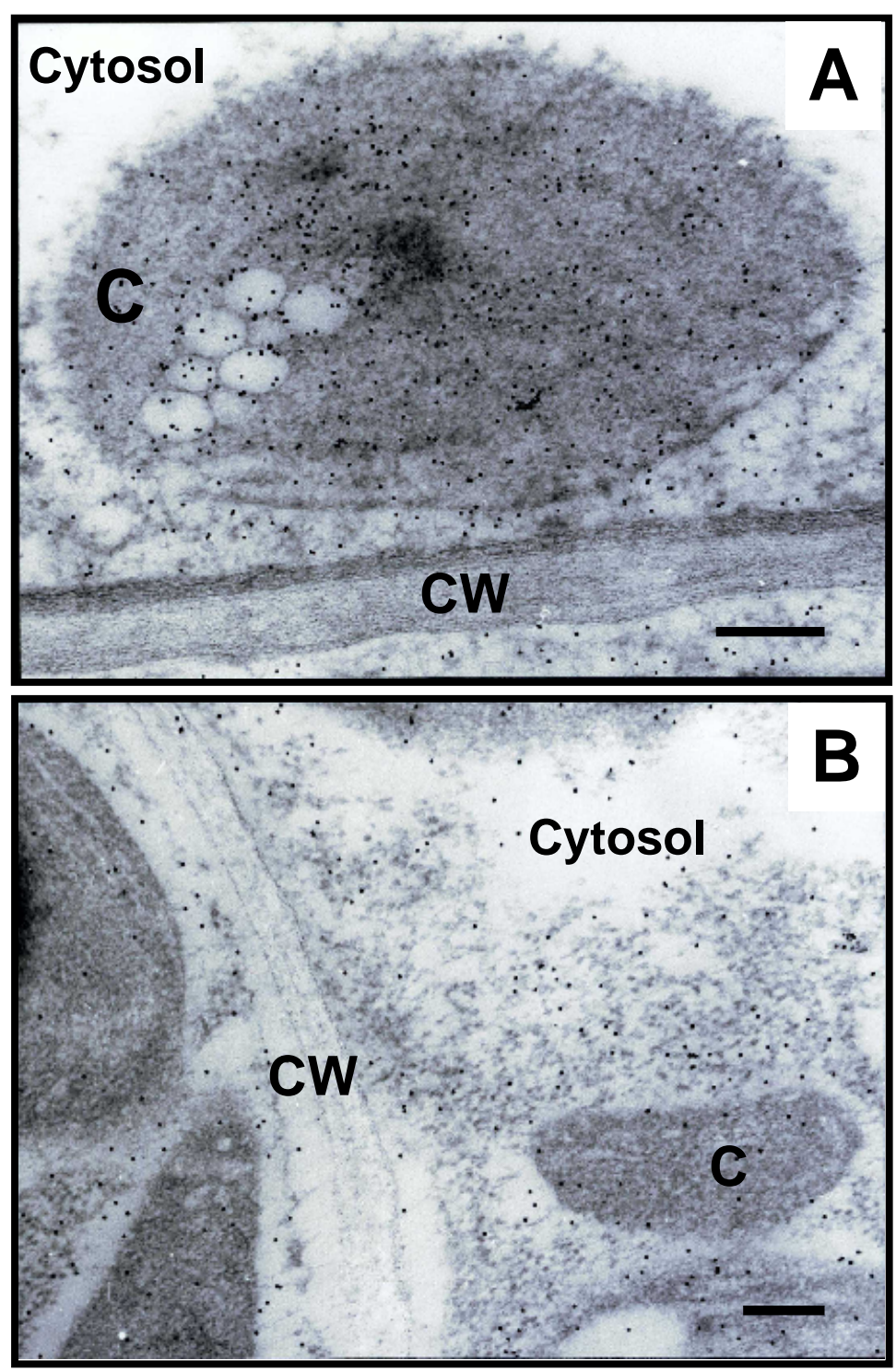

Figs. A, B. Immunolocalization of the endoglucanase EGIV-CBDII in transgenic tobacco leaf. A) Transgenic plant with the transit peptide. Numerous gold particles are present over the chloroplast. B) Transgenic plant without the transgenic peptide. Gold particles are more over the cytosol than over chloroplast. C: chloroplast; CW: cell wall. Scale bar; $200 \mathrm{~nm}$ 